\title{
RELAY SUBSET SELECTION IN COGNITIVE NETWORKS WITH IMPERFECT CSI AND INDIVIDUAL POWER CONSTRAINTS
}

\author{
Luis Blanco* and Montse Nájar ${ }^{\dagger}$ \\ ${ }^{*}$ Centre Tecnològic de Telecomunicacions de Catalunya (CTTC), Castelldefels, Spain. Email: 1blanco@cttc.es \\ ${ }^{\dagger}$ Universitat Politècnica de Catalunya (UPC), Barcelona, Spain. Email: montse.najar@upc.edu
}

\begin{abstract}
This paper considers the relay subset selection problem in an underlay cognitive network in which two secondary users communicate assisted by a set of $N$ potential relays. More specifically, this paper deals with the joint problem of choosing the best subset of $L$ secondary relays and their corresponding weights which maximize the Signal-toInterference-plus-Noise ratio (SINR) at the secondary user receiver, subject to per-relay power constraints and interference power constraints at the primary user. This problem is a combinatorial problem with a high computational burden. Nevertheless, we propose a sub-optimal technique, based on a convex relaxation of the problem, which achieves a nearoptimal performance with a reduced complexity. Contrary to other approaches in the literature, the secondary relays are not limited to cooperate at full power.
\end{abstract}

Index Terms - Multiple relay selection

\section{INTRODUCTION}

The recent emergence of the cognitive radio paradigm [1] is motivated by the rapid development of high data rate communication systems and the scarcity of spectrum resources. The aim of cognitive radio is to alleviate the inefficient utilization of limited spectrum resources in wireless communications by allowing unlicensed (secondary) users to access the spectrum originally allocated to licensed (primary) users. In cognitive radio networks, secondary users can access the licensed spectrum using three different approaches: underlay, overlay and interwave [1]. In the underlay approach, Secondary Users (SUs) are allowed to share the spectrum with the Primary Users (PUs) as long as the interference power level at the primary receiver is below a predefined threshold, a.k.a interference temperature. This problem is challenging, especially when the interference power constraint for the primary user is very stringent. This is due to the fact that the interference constraint limits the allowed transmit power of the secondary

The research leading to these results has received funding from the European Commission in the framework of the FP7 Network of Excellence in Wireless COMmunications NEWCOM\# (Grant agreement no.318306) and from the Catalan Government under grants 2014-SGR-60 and 2014-SGR1551 users and, consequently, the Quality of Service (QoS) of the secondary network. To address this issue, cooperative relaying among the secondary users has been considered as a potential solution to improve the QoS of the Secondary Network (SN) while respecting the interference threshold imposed by the PUs. In particular, distributed relay beamforming [2], is a powerful tool which has attracted especial interest in cognitive networks, e.g. in [3], [4], [5]. In this technique the relays of the cognitive network cooperate adjusting their transmission weights in order to form a beam to the secondary destination, mitigating the effect of the undesired bidirectional interferences between the primary and the secondary networks and improving the QoS at the secondary receivers.

A main challenge in designing wireless cooperative networks is how to effectively select the cooperative relay nodes. Most of the approaches in the literature are based on the selection of the best relay for the cooperation. Nevertheless, in adverse environments transmitting over a single relay may not be sufficient to achieve the desired QoS in the primary and the secondary networks and Multiple Relay Selection (MRS) problem has recently attracted interest in cognitive relay networks, e.g. [6-9]. In all these works each relay either cooperates with full transmission power or does not cooperate at all. Nonetheless, this approach is not optimal because, as is well known, the optimal beamformer results in relay powers that are not necessarily at their maximum allowable values [2]. Moreover, the approaches in [6]- [9] are based on the knowledge of the instantaneous Channel State Information (CSI) of the channels of the primary and the secondary networks. Unfortunately, as it is explained in [3], acquiring instantaneous CSI may be a difficult task in practical scenarios, specially for the interference channels, due to the incoordination between the primary and the secondary networks.

In this paper, we propose a new technique which deals with the problem of multiple relay selection in a two-hop cognitive relay network with individual power constraints at the secondary relays. More specifically, this paper addresses the joint problem of selecting the best subset of secondary relays and their corresponding weights which maximize the SINR at the secondary destination. This problem is addressed considering per-relay power constraints and keeping the interference to the primary network below a predefined threshold. In 
contrast to other methods in the literature, the exposed technique assumes the knowledge of the second-order statistics of the channels of the primary and the secondary network at the secondary network and the relays are not limited to cooperate with full power. Even though, the problem of choosing the best subset of $L$ relay nodes out of the set of $N$ potential relays, with per-relay constraints is a hard combinatorial problem, it can be relaxed into a convex one using sparsitypromoting norms. The approach considered herein is based on the use of the $l_{1}$-norm squared [10], a surrogate of the cardinality which promotes appearance of zeros in the beamformer vector, and achieves a near-optimal performance with a reduced computational complexity.

\section{PROBLEM FORMULATION}

Consider the cognitive radio system shown in Fig. 1 in which a primary and a secondary network coexist over the same spectrum band. The primary network consists of a transmitter-receiver pair, which are denoted by PU-TX and PU-RX, respectively, and in the secondary network a source (SU-S) sends its data to a secondary destination (SU-D) assisted by a set of $N$ secondary users, which can potentially act as relays. Each of the nodes in this scheme is equipped with a single antenna. Furthermore, the link between the SU-S and the SU-D is not considered due to large path loss. Let $\mathbf{f}=\left[f_{1}, \ldots, f_{N}\right]^{T}$ and $\mathbf{g}=\left[g_{1}, \ldots, g_{N}\right]^{T}$ represent the flat fading channels between SU-S and the relays and between the relays and SU-D, respectively. Since the Primary Network (PN) and the Secondary Network (SN) share the same band, we need to consider the mutual interference between them. Hence, denote by $\mathbf{f}_{p}=\left[f_{p 1}, \ldots, f_{p N}\right]^{T}$ and $\mathbf{g}_{p}=\left[g_{p 1}, \ldots, g_{p N}\right]^{T}$ the channels between PU-TX and the secondary relays and between the relays and PU-RX. In a similar way, $h_{p s}$ and $h_{s p}$ represent the interference links from PU-TX to SU-D and from SU-S to PU-RX, respectively.

As has been exposed in the Introduction, acquiring instantaneous CSI may be a difficult task in practical scenarios. To avoid the need to know instantaneous CSI, the channel coefficients of the cognitive system in Fig. 1 are modeled as random values. In this paper, similar to [2]- [4], it is assumed that the joint Second-Order Statistics (SOS) of the channel gains are available at the SN. This approach allows us to consider uncertainty in the channel models through introducing the covariance matrices of the channels.

The transmission process of the SN takes place in two successive time slots whereas the primary network is always transmitting. In the first time slot, the relays receive the signal transmitted by SU-S and the interference from the PU-TX. Assuming that $\sqrt{P_{s}} x_{s}$ (with $E\left\{\left|x_{s}\right|^{2}\right\}=1$ ) and $\sqrt{P_{p}} x_{p}^{(1)}$ (with $E\left\{\left|x_{p}^{(1)}\right|^{2}\right\}=1$ ) are the signals transmitted during the first slot by SU-S and PU-TX, the signal received at the $i t h$

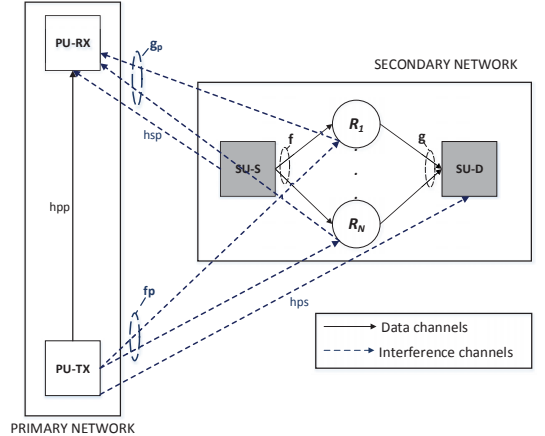

Fig. 1. Cognitive system

relay is given by

$$
r_{i}=\sqrt{P_{s}} f_{i} x_{s}+\sqrt{P_{p}} f_{p i} x_{p}^{(1)}+\nu_{i}
$$

being $P_{s}$ and $P_{p}$ the transmit powers of SU-S and PU-TX, respectively, and $\nu_{i}$ represents a zero-mean Additive White Gaussian Noise (AWGN) with known variance $\sigma_{\nu}^{2}$. In the second slot, the relays multiply the received signal by a complex weight and retransmit $t_{i}=w_{i} r_{i}$ to the SU-D. The received signal at the secondary destination in the second slot is

$$
\begin{aligned}
y_{d}= & \underbrace{\sqrt{P_{s}} \sum_{i=1}^{N} w_{i} f_{i} g_{i} x_{s}}_{\text {signal of interest }}+\underbrace{\sqrt{P_{p}} \sum_{i=1}^{N} w_{i} f_{p i} g_{i} x_{p}^{(1)}+\sqrt{P_{p}} h_{p s} x_{p}^{(2)}}_{\text {interference from PU-TX }} \\
& +\underbrace{\sum_{i=1}^{N} w_{i} g_{i} \nu_{i}+n_{s}}_{\text {Total noise }}
\end{aligned}
$$

where $x_{p}^{(2)}$ is the signal transmitted by PU-TX during the second slot and $n_{s}$ is the zero-mean AWGN at SU-D which has a variance $\sigma_{s}^{2}$. Meanwhile, at the same time slot, PU-RX receives the signal transmitted by PU-TX and the interference from the secondary relays. The interference term at the PU$\mathrm{RX}$ during the second time slot is given by

$$
y_{p}=\underbrace{\sqrt{P_{p}} \sum_{i=1}^{N} w_{i} f_{p i} g_{p i} x_{p}^{(1)}}_{\text {self-interference }}+\underbrace{\sqrt{P_{s}} \sum_{i=1}^{N} w_{i} f_{i} g_{p i} x_{s}+\sum_{i=1}^{N} w_{i} g_{p i} \nu_{i}}_{\text {interference of the SN }}
$$

Note that the transmit power of SU-TX has to be adjusted in order to fulfill the interference power constraint in the primary receiver in the first time slot. Therefore, $P_{s}$ is chosen as follows $P_{s}=\min \left\{P_{s}^{\max }, \frac{I_{t h}}{E\left\{\left|h_{s p}\right|^{2}\right\}}\right\}$, where $P_{s}^{\max }$ is the maximum transmit power of the secondary source and $I_{t h}$ is the maximum tolerable interference level at PU-RX. 


\subsection{Subset relay selection and SINR maximization under per-relay and interference power constraints}

Consider the joint problem of selecting the best subset of $L$ relay nodes for the retransmission, out of a set of $N$ potential secondary relays and their corresponding weights, which maximize the SINR at SU-D under per-relay power constraints. This problem has to be addressed keeping the interference below the maximum tolerable level at the PU-RX

$$
\begin{array}{ll}
\max _{\mathbf{w}} & \text { SINR } \\
\text { s.t. } & p_{i} \leq P_{i} \quad \forall i=1, \ldots, N \\
& \operatorname{card}(\mathbf{w})=L, P_{R} \leq I_{t h}
\end{array}
$$

where $\mathbf{w}=\left[w_{1} \ldots w_{N}\right]^{T}$ is the network beamforming vector and $\operatorname{card}(\cdot)$ denotes the cardinality operator which returns the number of non-zero coefficients of its argument. $P_{i}$ and $p_{i}$ are maximum allowable transmit power and the actual transmit power of the $i$ th relay, respectively, and $P_{R}$ is the interference power received at PU-RX caused by the SN.

Let us derive the mathematical formulation of this problem. Bearing in mind (2) and assuming that the symbols transmitted by PU-TX and SU-D are zero-mean independent, it is possible to show, after some straightforward but tedious manipulations, that the SINR is given by

$$
\operatorname{SINR}=\frac{\mathbf{w}^{H} \mathbf{A} \mathbf{w}}{\mathbf{w}^{H}(\mathbf{B}+\mathbf{C}) \mathbf{w}+\sigma_{T}^{2}}=\frac{\mathbf{w}^{H} \mathbf{A} \mathbf{w}}{\mathbf{w}^{H} \mathbf{D} \mathbf{w}+\sigma_{T}^{2}}
$$

being $\sigma_{T}^{2}=\sigma_{s}^{2}+P_{p} E\left\{\left|h_{p s}\right|^{2}\right\}$ and $\mathbf{A}=P_{s} E\left\{(\mathbf{f} \odot \mathbf{g})(\mathbf{f} \odot \mathbf{g})^{H}\right\}$. In this last expression $\odot$ stands for the Schur-Hadamard product. The matrices $\mathbf{B}$ and $\mathbf{C}$ are defined as follows

$$
\begin{aligned}
& \mathbf{B}=P_{p} E\left\{\left(\mathbf{f}_{p} \odot \mathbf{g}\right)\left(\mathbf{f}_{p} \odot \mathbf{g}\right)^{H}\right\} \\
& \mathbf{C}=\sigma_{\nu}^{2} \operatorname{diag}\left(E\left\{\left|g_{1}\right|^{2}\right\}, E\left\{\left|g_{2}\right|^{2}\right\}, \ldots, E\left\{\left|g_{N}\right|^{2}\right\}\right)
\end{aligned}
$$

We now derive the expression of interference power at PU-RX during the second slot. The interference is given by

$$
P_{R}=E\left\{\left|y_{p}\right|^{2}\right\}=\mathbf{w}^{H}(\mathbf{F}+\mathbf{G}+\mathbf{H}) \mathbf{w}=\mathbf{w}^{H} \mathbf{V} \mathbf{w}
$$

where $\mathbf{F}=\sigma_{\nu}^{2} \operatorname{diag}\left(E\left\{\left|g_{p 1}\right|^{2}\right\}, E\left\{\left|g_{p 2}\right|^{2}\right\}, \ldots, E\left\{\left|g_{p N}\right|^{2}\right\}\right)$, $\mathbf{G}=P_{s} E\left\{\left(\mathbf{f} \odot \mathbf{g}_{p}\right)\left(\mathbf{f} \odot \mathbf{g}_{p}\right)^{H}\right\}, \mathbf{H}=P_{p} E\left\{\left(\mathbf{f}_{p} \odot \mathbf{g}_{p}\right)\left(\mathbf{f}_{p} \odot \mathbf{g}_{p}\right)^{H}\right\}$

Finally, we need to formulate the individual power constraints at the relays in (3). The average transmit power of the $i t h$ relay is given by

$$
p_{i}=E\left\{\left|r_{i}\right|^{2}\right\}\left|w_{i}\right|^{2}=U_{i}\left|w_{i}\right|^{2}
$$

being $U_{i}=P_{s} E\left\{\left|f_{i}\right|^{2}\right\}+P_{P} E\left\{\left|f_{p i}\right|^{2}\right\}+\sigma_{\nu}^{2}$.

Using (5), (8) and (9), the optimization problem in (4) can be formally expressed as

$$
\begin{array}{ll}
\max _{\mathbf{w}} & \frac{\mathbf{w}^{H} \mathbf{A} \mathbf{w}}{\mathbf{w}^{H} \mathbf{D} \mathbf{w}+\sigma_{T}^{2}} \\
\text { s.t. } & U_{i}\left|w_{i}\right|^{2} \leq P_{i} \quad \forall i=1, \ldots, N \\
& \operatorname{card}(\mathbf{w})=L, \quad \mathbf{w}^{H} \mathbf{V w} \leq I_{t h}
\end{array}
$$

This is a computationally difficult problem which belongs to the class of NP-hard problems. To solve it an exhaustive search over all the possible sparsity patterns is needed. Since this search exhibits a high computational burden, this motivates the pursuit of efficient near-optimal methods.

\section{PROPOSED METHOD}

\subsection{Selection of the subset of secondary relays}

The problem in (10) is not convex. The goal of this section is to relax this problem into a convex one in order to obtain a new method with a lower computational burden. The classical approach in combinatorial optimization to relax cardinalityconstrained problems is to substitute the cardinality operator by the weaker but convex $l_{1}$-norm. The $l_{1}$-norm, which is defined as $\|\mathbf{w}\|_{1}=\sum_{i=1}^{i=N}\left|w_{i}\right|$, has a sparsifying effect that has long been observed in signal processing. Nonetheless, a different approach is considered herein. Similar to [10], the $l_{1}$-norm squared, denoted as $\|\mathbf{w}\|_{1}^{2}$, is considered instead. The $l_{1}$-norm squared is a sparsity-inducing norm that promotes the appearance of zeros in the final solution. Furthermore, it turns out that finding a convex approximation of (10) is easier when the problem is relaxed using $l_{1}$-norm squared. As is shown in the sequel, the relaxation of (10) with the $l_{1}$-norm squared naturally leads to semidefinite programming (SDP). Let us relax (10) replacing the cardinalty by the $l_{1}$-norm squared

$$
\begin{array}{ll}
\max _{\mathbf{w}} & \frac{\mathbf{w}^{H} \mathbf{A} \mathbf{w}}{\mathbf{w}^{H} \mathbf{D} \mathbf{w}+\sigma_{T}^{2}} \\
\text { s.t. } & U_{i}\left|w_{i}\right|^{2} \leq P_{i} \quad \forall i=1, \ldots, N \\
& \mathbf{w}^{H} \mathbf{V} \mathbf{w} \leq I_{t h} \\
& \|\mathbf{w}\|_{1}^{2} \leq \delta
\end{array}
$$

where $\delta$ is a positive parameter which controls the number of active relays. The discussion about how to effectively adjust this parameter to perform the selection of the subset of $L$ secondary relays will be presented in the following subsection. The problem in (11) is still NP-hard. This motivates the use of a semidefinite relaxation (SDR) to handle it. Let us rewrite the constraint (11d) in terms of $\mathbf{X}=\mathbf{w} \mathbf{w}^{H}$

$$
\|\mathbf{w}\|_{1}^{2}=\left(\sum_{i=1}^{i=N}\left|w_{i}\right|\right)^{2}=\mathbf{1}_{N}^{T}|\mathbf{X}| \mathbf{1}_{N}=\operatorname{Tr}\left\{\mathbf{1}_{N \times N}|\mathbf{X}|\right\}
$$

where $\mathbf{1}_{N}$ is a column-vector of ones of length $N,|\mathbf{X}|$ is the element-wise absolute value of $\mathbf{X}$ and $\mathbf{1}_{N \mathrm{x} N}$ is an $N \mathrm{x} N$ matrix with all one entries. By considering (12), the problem in (11) can be expressed as

$$
\begin{array}{ll}
\max _{\mathbf{X}} & \frac{\operatorname{Tr}\{\mathbf{A X}\}}{\operatorname{Tr}\{\mathbf{D X}\}+\sigma_{T}^{2}} \\
\text { s.t. } & X_{i i} \leq u_{i} \forall i=1, \ldots, N \\
& \operatorname{Tr}\left\{\mathbf{1}_{N \times N}|\mathbf{X}|\right\} \leq \delta ; \operatorname{Tr}\{\mathbf{V X}\} \leq I_{t h} \\
& \operatorname{rank}(\mathbf{X})=1 ; \mathbf{X} \succeq 0
\end{array}
$$


being $u_{i}$ the ith component of the vector $\mathbf{u}$ defined as $\mathbf{u}=\left[P_{1} / U_{1}, \ldots, P_{N} / U_{N}\right]^{T}$ and $X_{i i}$ the $(i, i)$ th element of $\mathbf{X}$. By dropping the rank constraint we obtain

$$
\begin{array}{cl}
\max _{\mathbf{X}} & \frac{\operatorname{Tr}\{\mathbf{A} \mathbf{X}\}}{\operatorname{Tr}\{\mathbf{D X}\}+\sigma_{T}^{2}} \\
\text { s.t. } & X_{i i} \leq u_{i} \forall i=1, \ldots, N \\
& \operatorname{Tr}\left\{\mathbf{1}_{N \times N}|\mathbf{X}|\right\} \leq \delta ; \operatorname{Tr}\{\mathbf{V X}\} \leq I_{t h} ; \mathbf{X} \succeq 0
\end{array}
$$

Due to the fractional structure of its objective (14a), this problem is quasi-convex. Fortunately, it can be reformulated as a SDP. Consider the following transformation of variables:

$$
\eta=\frac{1}{\operatorname{Tr}\{\mathbf{D X}\}+\sigma_{T}^{2}}, \quad \mathbf{Z}=\eta \mathbf{X}=\frac{\mathbf{X}}{\operatorname{Tr}\{\mathbf{D X}\}+\sigma_{T}^{2}}
$$

and rewrite (14) as

$$
\begin{array}{ll}
\max _{\mathbf{Z}, \eta} & \operatorname{Tr}\{\mathbf{A} \mathbf{Z}\} \\
\text { s.t. } & Z_{i i} \leq \eta u_{i} \forall i=1, \ldots, N \\
& \operatorname{Tr}\left\{\mathbf{1}_{N \times N}|\mathbf{Z}|\right\} \leq \eta \delta \\
& \operatorname{Tr}\{\mathbf{D Z}\}+\sigma_{T}^{2} \eta=1 \\
& \operatorname{Tr}\{\mathbf{V Z}\} \leq \eta I_{t h} ; \mathbf{Z} \succeq 0 ; \eta>0
\end{array}
$$

This problem is a SDP and can be efficiently solved (in polynomial time) using standard interior point solvers. If $\left(\mathbf{Z}^{*}, \eta^{*}\right)$ is the optimal solution of (16), then $\mathbf{X}^{*}=\mathbf{Z}^{*} / \eta^{*}$ is the solution of the problem in (14). The subset of secondary relays selected for the cooperation is determined as follows. The null elements of the diagonal of $\mathbf{Z}^{*}$ correspond to the nodes that should be left out in the retransmission stage, whereas the non-zero elements are the nodes chosen for the cooperation.

\subsection{Computation of the beamforming weights}

Once the $L$ relays are selected, the weights of these nodes need to be computed. They cannot be directly extracted from the solution of (16) because of the influence of the $l_{1}$-norm behind (16c). To compute the weights, we need to remove this constraint and the subset of inactive relays of this problem. Bearing this in mind, denote by $J \subseteq\{1, \ldots, N\}$ the subset of relays selected for the retransmission and by $\tilde{\mathbf{w}}=$ $\left[w_{J(1)}, \ldots, w_{J(L)}\right]^{T}$ their corresponding weights. To compute the weights we need to solve the next reduced-size problem

$$
\begin{aligned}
\max _{\tilde{\mathbf{Z}}, \eta} & \operatorname{Tr}\{\tilde{\mathbf{A}} \tilde{\mathbf{Z}}\} \\
\text { s.t. } & \tilde{Z}_{i i} \leq \eta \tilde{u}_{i} \quad \forall i=1, \ldots, K \\
& \operatorname{Tr}\{\tilde{\mathbf{D}} \tilde{\mathbf{Z}}\}+\sigma_{T}^{2} \eta=1 \\
& \tilde{\mathbf{Z}} \succeq 0, \operatorname{Tr}\{\tilde{\mathbf{V}} \tilde{\mathbf{Z}}\} \leq \eta I_{t h}, \eta>0
\end{aligned}
$$

where $\tilde{\mathbf{A}}, \tilde{\mathbf{D}}$ and $\tilde{\mathbf{V}}$ are submatrices of $\mathbf{A}, \mathbf{D}$ and $\mathbf{V}$ constructed by omitting the entries corresponding to the left-out relays and $\tilde{u}_{i}$ denotes the $i t h$ entry of the vector $\tilde{\mathbf{u}}$ which is constructed by selecting the subset of active relays from $\mathbf{u}$. In the same way, $\tilde{\mathbf{Z}}$ is a square matrix of size $L$ formed by the active rows and columns of $\mathbf{Z}$. Let us denote by $\left(\tilde{\mathbf{Z}}^{*}, \eta^{*}\right)$ the solution of (17). Due to the rank relaxation, $\tilde{\mathbf{Z}}^{*}$ might not be a rank-one matrix in general. When $\tilde{\mathbf{Z}}^{*}$ is rank one, the relaxation is tight and weights of the selected relays $\tilde{\mathbf{w}}$ can be directly obtained from the eigendecomposition of $\boldsymbol{\Psi}=\tilde{\mathbf{Z}}^{*} / \eta^{*}$. If $\operatorname{rank}\left(\tilde{\mathbf{Z}}^{*}\right)>1$, an approximate solution can be obtained using randomization [2].

\subsection{Adjustment of the value of $\delta$}

The cardinality of the solution of (16) is controlled by the tuning parameter $\delta$. In particular, the number of selected relays is reduced when $\delta$ is decreased. The goal of this subsection is to describe a method for the appropriate selection of this parameter. The proposed technique relies on a binary search over $\delta$ that successively decreases the cardinality of the solution until a solution with $L$ active entries is obtained. Recall (11d), i.e., $\|\mathbf{w}\|_{1}^{2} \leq \delta$, and consider the following bounds on the $l_{1}$-norm squared [11]

$$
\|\mathbf{w}\|_{2}^{2} \leq\|\mathbf{w}\|_{1}^{2} \leq L\|\mathbf{w}\|_{2}^{2}
$$

where $\|\mathbf{w}\|_{2}^{2}$ denotes the square of the Euclidean norm. This expression will be used to find an initial value of $\delta$ for the binary search that will be denoted by $\delta_{\max }$. From the right hand inequality is clear that if an upper bound on the Euclidean norm can be determined, it can be used to compute $\delta_{\max }$. To obtain this bound consider the problem in (17) assuming that all the relays are active, i.e., consider $\tilde{\mathbf{A}}=\mathbf{A}, \tilde{\mathbf{V}}=\mathbf{V}$, $\tilde{\mathbf{D}}=\mathbf{D}$ and $\mathbf{u}=\tilde{\mathbf{u}}$, and let $\mathbf{w}^{(0)}$ be the optimal beamformer obtained from the solution of this problem. From (11d) and (18), it is clear that $\delta=L\left\|\mathbf{w}^{(0)}\right\|_{2}^{2}$ ensures that at least $L$ relays will be active. This is due to the fact that by decreasing $\delta$ to obtain $L$ active relays, one is also decreasing $\|\mathrm{w}\|_{1}^{2}$ and, consequently, $\|\mathbf{w}\|_{2}^{2}$. Unfortunately, $\delta=\delta_{\max }$ often enforces solutions with more than $L$ active entries. In this case, we need to decrease $\delta$ by considering a binary search until a solution with the desired cardinality is obtained. This search requires solving (16) for different values of $\delta$. Nonetheless, the number of required SDP in this binary search is far less than in the exhaustive search which requires solving $\left(\begin{array}{c}N \\ L\end{array}\right)$ problems of type (17). This will be analyzed later with numerical results. The whole technique is described in Algorithm 1.

\section{SIMULATION RESULTS}

In this section, we provide numerical simulations to show the performance of the proposed algorithm. To solve the SDP problems CVX [12] is used. The considered $\mathrm{SN}$ is composed of a source with $P_{s}^{\max }=3 \mathrm{dBW}$ and $N=12$ potential relays whose individual power constraints are uniformly given by $P_{i}=P_{s}^{\max }$. In the PN, PU-TX transmits with $P_{p}=3$ dBW. The channels $\left\{f_{i}\right\}_{i=1}^{N},\left\{g_{i}\right\}_{i=1}^{N},\left\{f_{p i}\right\}_{i=1}^{N},\left\{g_{p i}\right\}_{i=1}^{N}, h_{s p}$ and $h_{p s}$ are assumed to be independent from each other and 


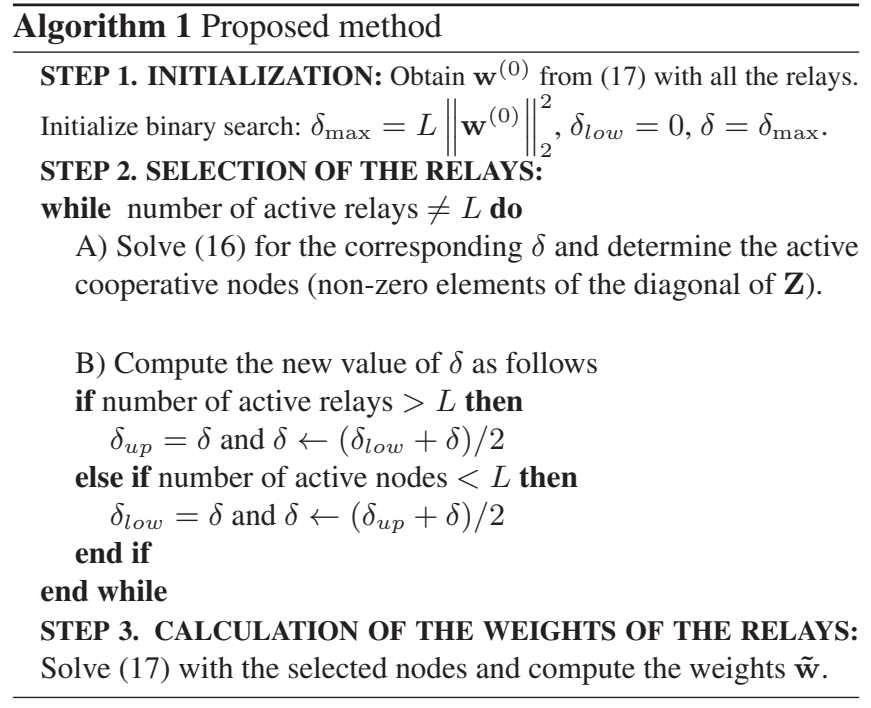

the parameters $P s, \mathbf{A}, \mathbf{D}$ and $\{U\}_{i=1}^{N}$ have been generated assuming that the second-order statistics of these channels are known. The results are obtained after averaging 100 realizations, in each of the trials the means of the channels are generated randomly as $\mathcal{C N}(0,1)$ and the variances of these channels are generated following $\frac{1}{2} \mathcal{X}^{2}(2)$, where $\mathcal{X}^{2}(2)$ denotes the chi-square distribution with two degrees of freedom. $I_{t h}$ is set to $0 \mathrm{dBW}$ and $\sigma_{s}^{2}=\sigma_{\nu}^{2}=-3 \mathrm{dBW}$. Fig. 2 shows the SINR achieved at SU-D as a function of the number of selected relays. The performance of the proposed technique is very close to the one achieved with the exhaustive search and clearly outperforms the random selection of the subset. Moreover, our method has a computational complexity that is far less than the one of exhaustive search. The mean number of SDPs that needs to be solved in proposed algorithm is less than 8 for any value of $L$ (less than 7 for the selection of the subset plus one for the computation of the optimal weights). The worst case was obtained for $L=6$ and has required the computation of 13 SDPs, far less than the exhaustive search that requires $\left(\begin{array}{c}12 \\ 6\end{array}\right)=924$ SDPs.

Fig. 3 shows the maximum achievable SINR at SU-D as a function of power constraint at the primary receiver $I_{t h}$ when 5 relays are selected. Again the performance of the proposed algorithm is very close to the SINR-optimal performance.

\section{CONCLUSIONS}

We have investigated the multiple relay selection problem in underlay cognitive networks. A new sub-optimal method with a reduced complexity has been proposed for selecting the subset of secondary relays in a two-hop cognitive network with per-relay power constraints. This problem is addressed keeping the interference to the primary network below the maximum tolerable level.

\section{REFERENCES}

[1] Beibei Wang and K. J. Ray Liu, "Advances in cognitive radio networks: A survey.," J. Sel. Topics Signal Processing, vol. 5, no. 1, pp. 5-23, 2011 .

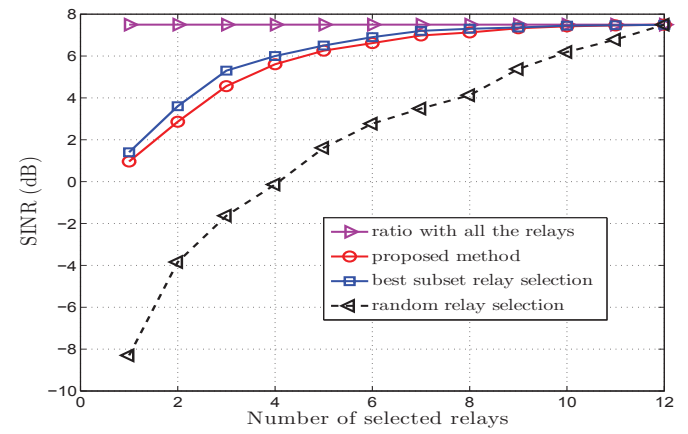

Fig. 2. Achieved SINR at SU-D as a function of L

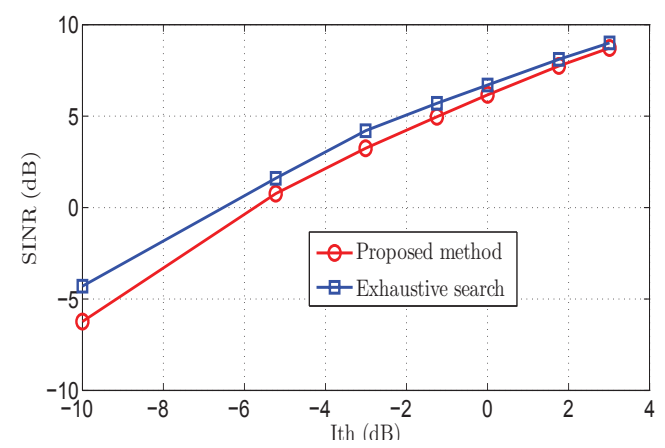

Fig. 3. Achieved SINR as a function $I_{t h}$

[2] V. Havary-Nassab, S. Shahbazpanahi, A. Grami, and Zhi-Quan Luo, "Distributed beamforming for relay networks based on second-order statistics of the channel state information," Trans. Sig. Proc., vol. 56, no. 9, pp. 4306-4316, Sept. 2008.

[3] Amir Piltan and Soheil Salari, "Distributed beamforming in cognitive relay networks with partial channel state information.," IET Communications, vol. 6, no. 9, pp. 1011-1018, 2012.

[4] S.H. Safavi, M. Aredebilipour, and S. Salari, "Relay beamforming in cognitive two-way networks with imperfect channel state information.," IEEE Wireless Comm. Letters, vol. 1, no. 4, pp. 344-347, 2012.

[5] A. Alizadeh and H. R. Bahrami, "Optimal distributed beamforming for cooperative cognitive radio systems," in in Proc. 77th IEEE Vehicular Technology Conference (VTC Spring), Dresden, Germany, 2013.

[6] J. Xu, H. Zhang, D. Yuan, Q. Jin, and C. Wang, "Novel multiple relay selection schemes in two-hop cognitive relay networks.," IEEE Int. Conf. on Communications and Mobile Computing, pp. 307-310, 2011.

[7] M. Naem, D. Lee, and U. Pareek, "An efficient multiple relay selection scheme for cognitive radio systems," in Proc. IEEE ICC, 2010.

[8] Y. Li and A. Nosratinia, "Spectrum-sharing capacity enhancement with distributed relaying," in Proc. IEEE ICC, pp. 2197-2201, 2012.

[9] Jiali Xu, Haixia Zhang, and Dongfeng Yuan, "A multiple relay selection scheme in cognitive relay network," vol. 139, pp. 33-39, 2012.

[10] O. Mehanna, N.D. Sidiropoulos, and G. B. Giannakis, "Multicast beamforming with antenna selection," in Proc. 13th SPAWC, 2012.

[11] N. Bourbaki, H.G. Eggleston, and S. Madan, Topological Vector Spaces, Springer-Verlag, 1987.

[12] "CVX: Matlab software for Disciplined Convex Programming," http://www. cvxr. com/cvx/. 